\title{
Volumetric Plasma Discharge in a Coaxial Electrode Configuration Using Repetitively Pulsed Nanosecond Discharges
}

\author{
Ravichandra R. Jagannath*, Kyle Newnam ${ }^{\dagger}$, Paul W. Stockett ${ }^{\ddagger}$, Puyuan Wu $^{\S}$, Sally P. M. Baneף \\ Purdue University, West Lafayette, Indiana-47907 \\ M. Razi Nalim" \\ Indiana University Purdue University Indianapolis, Indiana-46202
}

\begin{abstract}
Transient plasma discharges can be created in different electrode geometries and the use of a coaxial electrodes can assist in initiating ignition at multiple points at the same time to create volumetric ignition. The current study investigates discharge formation in a coaxial electrode in quiescent, atmoshpheric and non-reacting conditions. This is the first systematic study to understand the behavior of such a discharge as a function of different pulse parameters like pulse width (40-110 ns), repetition frequency (1-50 $\mathrm{kHz})$ and input voltage (14-20 kV). Additionally, the polarity of the central electrode was changed between positive and negative. An intensified ccd camera was used to visualize the discharge formation. The exposure of the camera is set to capture 500 discharges in a single frame. The discharges were found to behave differently for positive and negative polarity discharges. The positive polarity discharge tends to form a strong arc and spins around the outer cylinder which is confirmed using a high speed camera. The negative polarity discharges form a uniform streamer discharge for most of the pulse parameters. The current study has provided an initial understanding of the dynamics of plasma discharges in a coaxial electrode.
\end{abstract}

\section{Introduction}

Ignition delay time plays a vital role in combustion and propulsion systems, including both steady flow devices such as gas turbine engines and unsteady flow devices such as automotive engines, pulse detonation engines, rotating detonation engines, and wave rotors. Different strategies such as stratification of the fuel/air mixture, ${ }^{1}$ introduction of a turbulent hot jet for ignition, ${ }^{2}$ and efficient injection methods have been attempted to reduce the ignition delay, but it still remains in the millisecond range. A more recent approach to reducing ignition delay is ignition using transient, non-equilibrium plasmas. This method has proven to be more efficient compared to traditional spark ignition, ${ }^{3-5}$ which uses a thermal form of plasma to create a pocket of hot gas to initiate combustion. In the case of non-equilibrium plasma ignition, a nanosecond-scale electrical discharge results in atoms and molecules that are excited to higher electronic, vibrational, and rotational states in which they remain for a few tens or hundreds of nanoseconds. The presence of these

\footnotetext{
* Graduate Research Assistant, School of Aeronautics and Astronautics, 701 W.Stadium Avenue, West Lafayette, Indiana47907, AIAA Student member, rjagann@purdue.edu.

${ }^{\dagger}$ Graduate Research Assistant, School of Aeronautics and Astronautics, 701 W.Stadium Avenue, West Lafayette, Indiana47907, AIAA Student member, knewnam@purdue.edu.

$¥$ Undergraduate Research Assistant, School of Aeronautics and Astronautics, 701 W.Stadium Avenue, West Lafayette, Indiana-47907, AIAA Student member, pstocket@purdue.edu.

$\S$ Graduate Research Assistant, School of Aeronautics and Astronautics, 701 W.Stadium Avenue, West Lafayette, Indiana47907, AIAA Student member, wu912@purdue.edu.

ฯ Assistant Professor, School of Aeronautics and Astronautics, 701 W.Stadium Avenue, West Lafayette, Indiana-47907, AIAA member, sbane@purdue.edu.

" Professor,Department of Mechanical Engineering, 723 W. Michigan St., Indianapolis. IN 46202-5160, AIAA Associate Fellow, mnalim@iupui.edu
}

This is the author's manuscript of the article published in final edited form as: Jagannath, R., Newnam, K., Stockett, P., Wu, P., Bane, S. P., \& Nalim, M. R. (2017). Volumetric Plasma Discharge in a Coaxial Electrode Configuration Using Repetitively Pulsed Nanosecond Discharges. In 55th AIAA Aerospace Sciences Meeting. American Institute of Aeronautics and Astronautics. https://doi.org/10.2514/6.2017-1974 
excited species can promote chain-branching reactions which lead to ignition. There are two phases in plasma ignition, the first being a non-thermal phase where the plasma discharge provides a large volume of excited species and the second being a thermal phase that provides the exothermic heat release.

The application potential of non-equilibrium plasma for ignition and combustion has increased over the past decade. The advent of high-voltage and high-repetition rate nanosecond pulsers has enabled the use of ultra-short duration, high-frequency plasma discharges for aerodynamic flow control ${ }^{6-8}$ and combustion enhancement..$^{3,9-12}$ Spark plasmas have been used for more than a century as ignition devices in a wide array of combustion systems. A spark discharge produces a small region of high temperature plasma, which then creates a pocket of hot gas to initiate combustion. The total energy input is much higher than that required for ignition and a portion of the energy is lost to the electrodes through heat conduction. Since the high-temperature gas is localized in a small region, the presence of a combustible mixture at this location is critical. In addition, typical ignition delays using traditional spark discharges are on the order of tens of milliseconds which is not ideal for high-speed or highly transient combustion devices. Spark plasmas used in conventional spark ignition systems are typically generated using high voltage pulses with durations of 10s or 100s of microseconds. Plasmas generated by nanosecond-scale duration discharges have significantly different characteristics. The high electric field combined with short duration time inhibits arc transition and generates a plasma in thermal non-equilibrium where only the electrons are at extremely high temperature. Collisions between these electrons with very large kinetic energy and fuel/oxidizer molecules result in production of active radicals and excited species in streamer channels through electron impact dissociation, excitation and ionization. ${ }^{13}$ The generation of these excited and active species alters the chemical kinetics and can lead to more efficient radical production, promotion of chain-branching reactions, and ultimately a shorter ignition delay. $^{4}$

Much of the previous research on plasma-assisted ignition has used pin-to-pin electrodes resulting in single point ignition. If the electrode configuration is designed to produce multiple streamers, it could potentially result in a larger volume of active radicals and multiple ignition locations. There have been only a few studies on multi-point ignition using transient plasmas. Singleton et.al. investigated multi-point ignition of $\mathrm{C}_{2} \mathrm{H}_{4}$-air mixtures at atmospheric pressure using nanosecond pulsed plasmas. ${ }^{4}$ The electrode configuration used a stainless steel rod as the anode and stainless steel tube as the cathode to generate a volume of streamers between the rode and tube as shown in Figure 1. It was reported that ignition was initiated at multiple locations, at the base of the streamers where the electric field and therefore the concentration of active radicals were largest. The results also showed that the ignition delay was reduced by a factor of half for the transient plasma system compared to spark ignition as shown in Figure 2. The multi-point ignition concept was also demonstrated in single-cylinder engine and compared to spark ignition by Shiraishi and co-workers. ${ }^{10}$ Like in the study by Singleton et.al., a threaded rod was used as the anode and a cylinder was used as the ground electrode to generate multiple discharge filaments. It was reported that 6 ms after ignition, the flame had grown to a diameter of $93 \mathrm{~mm}$ with the transient plasma system versus $74 \mathrm{~mm}$ with the spark. In addition, the experiments showed a decrease in unburnt $\mathrm{HCs}$ and $\mathrm{CO}$ emission with the use of nanosecond plasma ignition. Nanosecond pulsed surface dielectric barrier discharges (ns-SDBD) have also been used to achieve multi-point ignition in a rapid compression machine. Tests were performed for a variety of different fuels and at elevated pressures from 17 to 40 bar, and a reduction in ignition delay from hundreds to tens of milliseconds was obtained using the ns-DBD. ${ }^{14,15}$ However, these experiments were conducted at high initial temperatures where it is easier to to achieve electrical breakdown in the gas, which is more difficult at elevated pressures. Laux and colleagues at Ecole Centrale Paris have investigated the use of nanosecond repetitively pulsed (NRP) plasmas in a pin-annular electrode configuration for controlling combustion instability and stabilizing flames. ${ }^{16,17}$ In more recent work, the influence of air flow and acoustic effects on the plasma in the pin-annular electrode configuration was investigated. ${ }^{18}$ To the author's knowledge there has been only one study where the pin electrode is axially separated from the annulus. In this work, the authors demonstrated that a pin electrode separated from a semi circular electrode performs better than pin-to-pin electrodes in terms of ignition delay. ${ }^{19}$

In the experiments performed using coaxial electrodes, the central electrode is usually chosen to be positively charged and the outer ring or cylinder is grounded. In this arrangement, a nanosecond scale high voltage pulse creates a cathode-directed streamer. Conversely, if a pulse of negative polarity is applied to the central electrode, it can create anode-directed streamers. The two types of streamers have been found to behave differently due to photo-ionization and electric field direction. The anode-directed streamer fades and disappears halfway through the gap as shown in Figure 3, whereas the cathode-directed streamer has been 


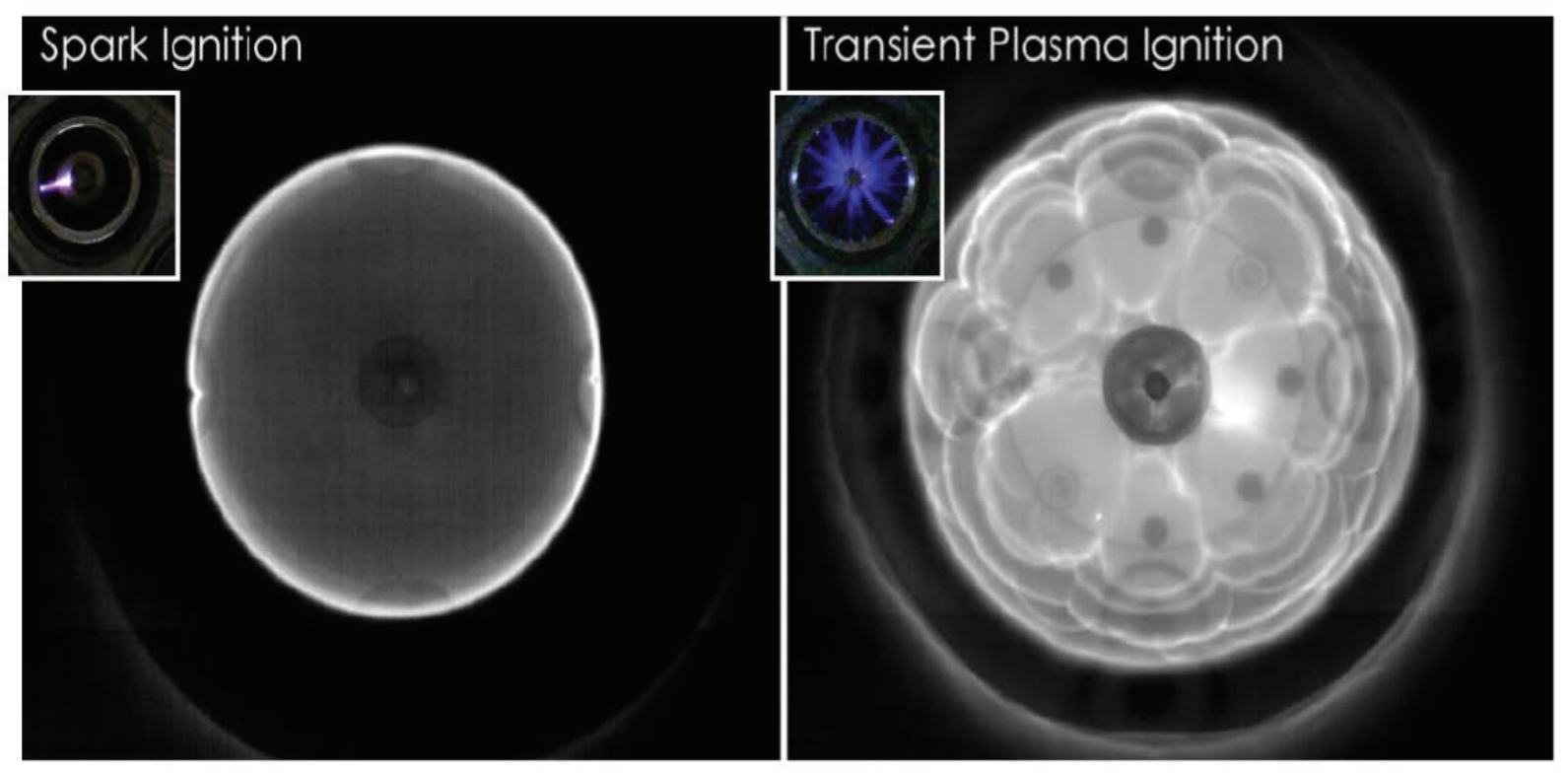

Figure 1. Flame propagation using spark ignition and transient plasma ignition ${ }^{4}$

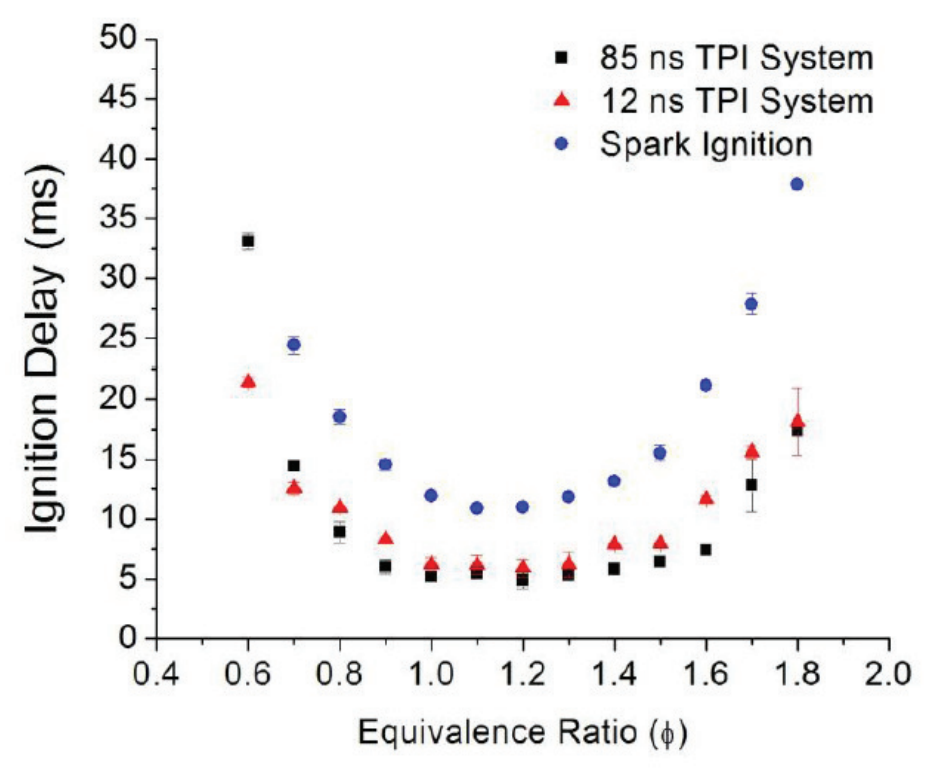

Figure 2. Ignition delay using transient plasma ignition with 12 ns and 85 ns pulse duration compared with traditional spark ignition ${ }^{4}$ 
found to slow down to a constant speed as the streamer propagates through the gap, but unlike the anode directed streamer the cathode directed streamer actually reaches the cathode. ${ }^{20}$ In a similar analysis on plasma jets, ${ }^{21}$ anode-driven (positively excited) and cathode-driven (negatively excited) atmospheric plasma jets were observed using fast imaging. It was found that the current measured in cathode-driven jets was half of the current measured for anode-driven jets, and thus the plasma emission was weaker for the cathodedriven jet. In addition, the anode-driven plasma plume travels three times longer than cathode-driven plume. The authors postulate that the different plume behaviors are due to the difference in direction of the transient electric field from the high voltage pulse and the field formed between propagating ionization fronts and the charge-accumulated dielectric surface at the nozzle exit.
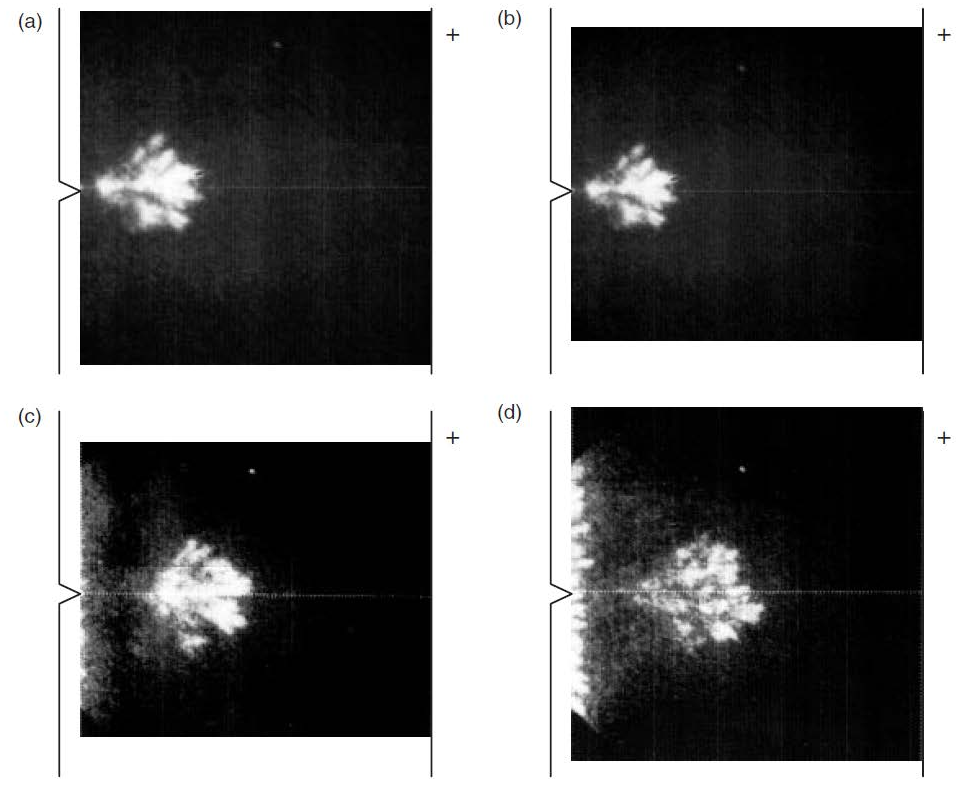

Figure 3. Sequence of shutter photographs of anode directed streamers in an atmospheric pressure $\mathbf{N}_{2}$ filled gap. Applied voltage is $123 \mathrm{kV}$, shutter is open for $10 \mathrm{~ns}$ and the photos are (a) 40, (b) 50 , (c) 60 and (d) 70 ns after the applied voltage pulse ${ }^{20}$

Shiraishi et.al. in their study of volumetric ignition showed that the plasma formation in coaxial electrodes transition from streamer to arc at different discharge voltages at different pressures as shown in Figure $4 .^{10}$ At atmospheric pressure, with a single pulse the transition was at $10 \mathrm{kV}$. In a similar experiment performed by Heitz et.al., the transion plot for plasma formation in a flow with a high repetition frequency of $30 \mathrm{kHz}$ is shown in Figure $5 .{ }^{18}$ At high repetition frequency, the transition happens between 7 and $8 \mathrm{kV}$. At lower repetition frequencies, this trend can shift upwards. This is because, the use of high repetition frequency allows lower breakdown voltages due to history effects. The input voltage was varied in the current work to account for this transition and future work will look into pressure effects.

The focus on the current work is to further investigate the plasma formed by nanosecond repetitively pulsed (NRP) discharges in coaxial electrode configurations. The tests are conducted in quiescent air at room temperature and atmospheric pressure; later work will examine the influence of ambient pressure and flow velocity. Increased understanding of these plasmas and the effects of various parameters (e.g. pulse peak voltage, repetition rate, electrode gap size, etc.) is a critical step towards application of such plasmas to ignition and combustion control. In the current work, the pulse peak voltage, pulse duration, and pulse repetition frequency (PRF) is varied to investigate the effect on the plasma formation in a coaxial electrode configuration. In addition, the effect of changing the polarity of the center electrode is explored. The plasma characteristics are investigated using ICCD images of 500 consecutive pulses, which is a time scale consistent with typical ignition and combustion processes.

Images of the plasma obtained when the center electrode was at negative potential (anode-directed) are compared with images when the center electrode was at positive potential (cathode-directed). The behavior is similar to what was observed previously for cathode- and anode-directed streamers. With the 


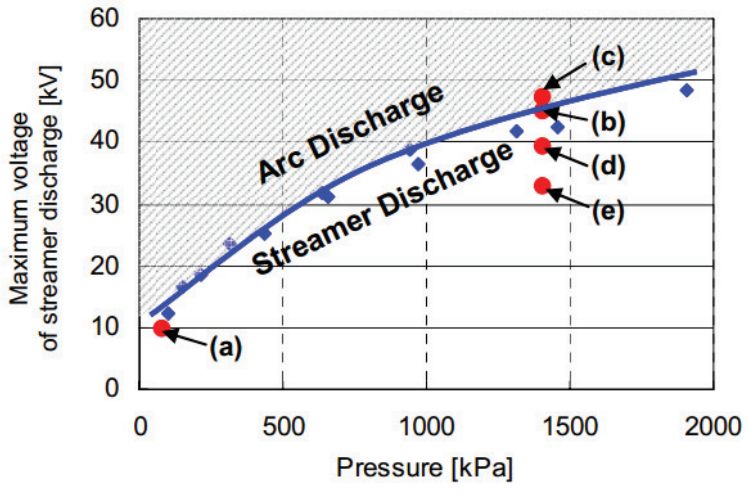

Figure 4. Streamer to arc transition trend as a function of pressure ${ }^{10}$

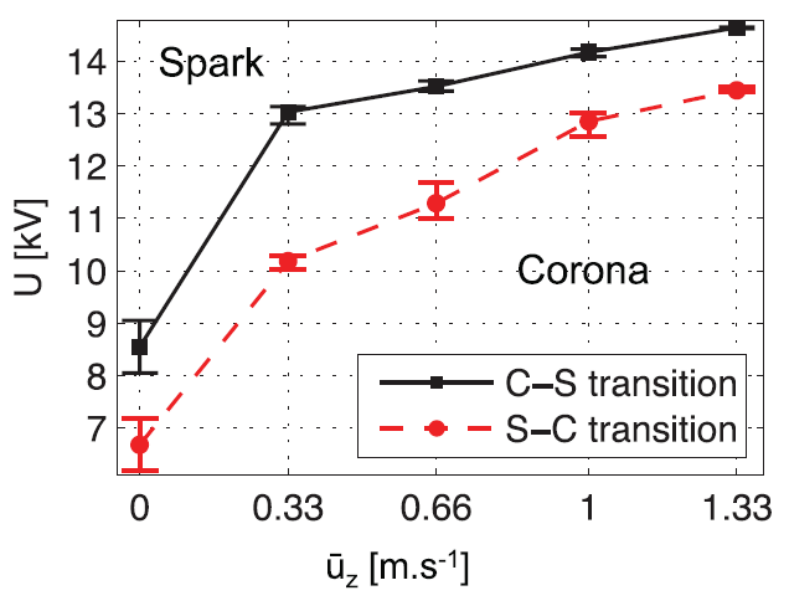

Figure 5. Streamer to arc transition trend as a function of flow velocity at atmospheric pressure ${ }^{18}$

center electrode at a positive potential, a discharge filament would form at one location about the electrode circumference and rotate about the axis of the cylinder when the pulser was driven at higher frequencies, while a more uniform discharge was formed in the opposite case (negative polarity). The interesting behavior of the positive-polarity discharge was further confirmed through visualization using a high-speed camera. This behavior of the discharge is important in the case of volumetric ignition. The current paper will first describe the experimental setup and equipment used, followed by intensified ccd images at different pulse parameters to qualitatively understand the difference observed in the two configurations and the configuration that may be beneficial for volumetric ignition.

\section{Experimental Setup}

A schematic of the experimental setup is shown in Figure 6. A 1 inch diameter stainless steel pipe is used for the outer cylinder electrode and 1/8" tungsten rod is used for the inner electrode. Both the electrodes are machined to minimize surface roughness and are cleaned with iso-propyl alcohol before every experiment. The electrodes are placed on opposite ends of an acrylic mount that is machined precisely so the two electrodes are concentric. In the current tests, the tip of the center electrode is aligned with the end of the cylinder electrode to produce plasma in a single plane; the axial distance between the electrodes is yet another parameter that can be varied to influence the plasma and will be the subject of a future study.

The high voltage pulses are generated using a custom-built NSP-3300-20-F nanosecond pulser from Eagle Harbor Technologies. The pulse parameters can be varied, with peak voltage up to $20 \mathrm{kV}$, pulse duration from $20 \mathrm{~ns}$ to $110 \mathrm{~ns}$, and pulse repetition frequency (PRF) up to $400 \mathrm{kHz}$. The pulser is powered by a TDK-Lambda power supply that can operate between 0 and $600 \mathrm{~V}$ DC. The pulser output scales linearly with the power supply input voltage, where $0-600 \mathrm{~V}$ on the power supply is equivalent to $0-25 \mathrm{kV}$ at the output; however, the resistive load at the pulser output restricts the voltage to a maximum of $20 \mathrm{kV}$. The output of the pulser is floating and the potential is symmetric with respect to ground. Therefore, during a pulse one electrode is at positive potential while the second electrode is at negative potential. The first set of experiments were performed with the center electrode at positive potential and the second set had the center electrode at negative potential. The pulser is triggered using a delay generator and a fiber optic transmitter. Two Tektronix P6015A high voltage probes are used to make a differential measurement of the voltage waveform. One probe is connected to the anode and the other to the cathode, and the ground cables of the two probes are connected. Therefore, the difference in the readings between the two probes gives the voltage across the gap. The discharge current will be measured in future studies to aid in interpretation of the discharge behavior and to enable discharge energy calculations.

A Princeton instruments PI-MAX4 1024i intensified CCD camera is used to image the discharge formation. The intensifier gate width is set to capture a series of 500 pulses in a single image to visualize the overall plasma development. In each experiment a burst of 1500 pulses is used, where the first 1000 pulses in 
the train are used to establish a "steady-state" plasma for all pulser parameters and the last 500 pulses are used for imaging. A signal generator is used to trigger both the pulser and the camera. The camera trigger has a delay $\Delta t$ with respect to the pulser signal to account for the delay between the pulser trigger and the discharge formation and the time required for the first 1000 pulses. The delay for the camera trigger is 1 , 0.1 , and 0.02 seconds for pulse repetition frequencies of 1,10 , and $50 \mathrm{kHz}$, respectively. The exposure time on the camera is set to 500,50 , and $10 \mathrm{~ms}$ for 1,10 , and $50 \mathrm{kHz}$, respectively to capture 500 consecutive pulses.

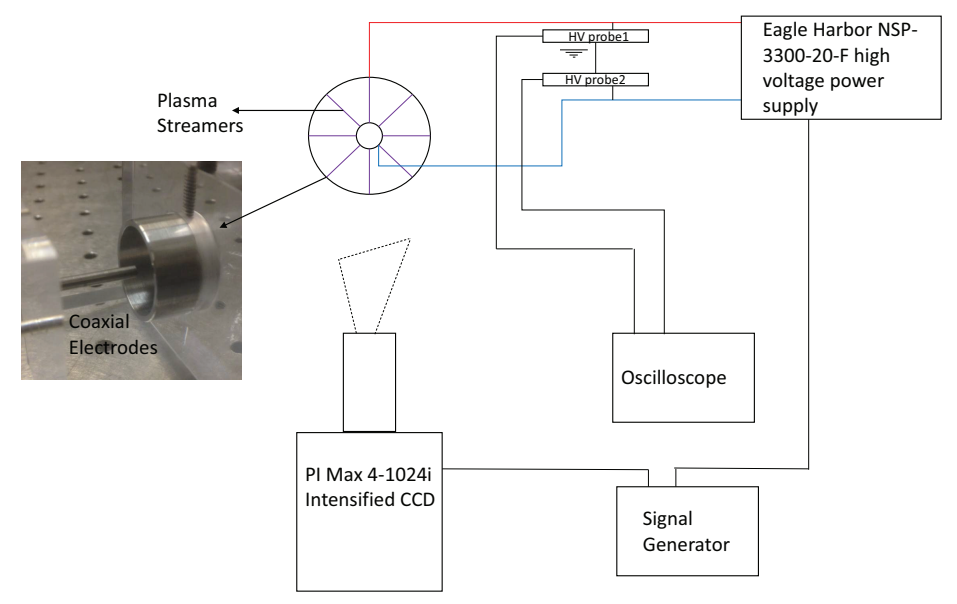

Figure 6. Schematic of the experimental setup. Coaxial electrodes used are also shown

\section{Results}

The main goal for the development of the ignition system in the current work is to achieve multi-point ignition with minimal ignition delay. Multi-point ignition depends on formation of multiple plasma streamers, where the base of each streamer is predicted to serve as an ignition site. These multiple discharges between electrodes create a region of volumetric plasma, providing a higher concentration of active radicals that can promote ignition and combustion. Therefore, it is important to design an electrode configuration that results in formation of volumetric plasma and to first understand the plasma dynamics in different non-reacting flow conditions .

In the current work, the plasma formation in a concentric electrode configuration is studied using a range of pulse peak voltage, pulse duration, and pulse repetition frequency. The effect of the pulse polarity is also investigated. While such plasmas have been shown to be promising for plasma-assisted ignition ${ }^{4,10}$ and combustion, ${ }^{16,17}$ the current study is the first systematic investigation of these plasmas and effects of varying pulse parameters. Four different peak pulse voltages were considered: approximately 14.6, 16.7, 18.75, and $20.8 \mathrm{kV}$. At each of these voltages, three different pulse duration (40,60, and $110 \mathrm{~ns}$ ) and three different pulse repetition frequencies $(1,10$, and $50 \mathrm{kHz})$ were tested. The full set of pulse parameter combinations investigated in the current work is given in Table 1. For each set of pulse parameters (peak voltage, duration, and PRF), discharges were studied for both negative and positive polarity of the central electrode. Images of 500 consecutive pulses were obtained for each set of pulse parameters and two polarities and are shown in Figures 8-15. The outer cylinder is marked with a red circle to get an understanding of streamer travel distance.

When the central electrode is at negative polarity (Figure 8) and at $1 \mathrm{kHz} \mathrm{PRF}$, the discharge appears quite uniform with diffuse discharge channels spaced at even intervals around the electrode circumference. There is little change in the discharge appearance when the pulse duration is increased from 40 to $110 \mathrm{~ns}$. The plasma mode is similar for $10 \mathrm{kHz}$ PRF, though the discharge channels appear a bit more constricted and the plasma emission intensity increases significantly as the pulse duration increases. The discharge propagation distance is also smaller for $10 \mathrm{kHz}$ vs. $1 \mathrm{kHz}$, except for the case with $110 \mathrm{~ns}$ pulse duration. At the highest PRF $(50 \mathrm{kHz})$ the discharge channels form distinct streamers and both the plasma propagation distance and the emission intensity decrease drastically. The drop in emission intensity forced the use of 
intensifier gain on the camera and therefore saturating some of the images. To maintain consistency, the number of pulses imaged was limited to 500, but in future work a larger number of pulses may be used to increase the total emission. As the voltage is increased, the negative polarity discharge transitions to an arc and behaves like the positive polarity discharge for a few pulse parameters as shown in $50 \mathrm{kHz}$ for Figures 10 and 12 and most of Figure 14. The positive polarity discharge on the other hand forms an arc discharge for most of the pulse parameters. At low PRF, it starts off as a single arc that spins about the axis and at higher PRFs, a higher number arcs coalesce together to form an arc at a random spot on the outer cylinder. The fact of spinning discharge was confirmed through the use of a high speed camera and the images are shown in Figure 7 . The discharge was created with a PRF of $2 \mathrm{kHz}$ and the high speed camera was operated at 10000 frames per second.The high speed video showed that when there is a positive polarity discharge, there is one strong arc and several other weak ones. The strong arc is seen rotating by more than $90 \circ$ in Figure 7 .
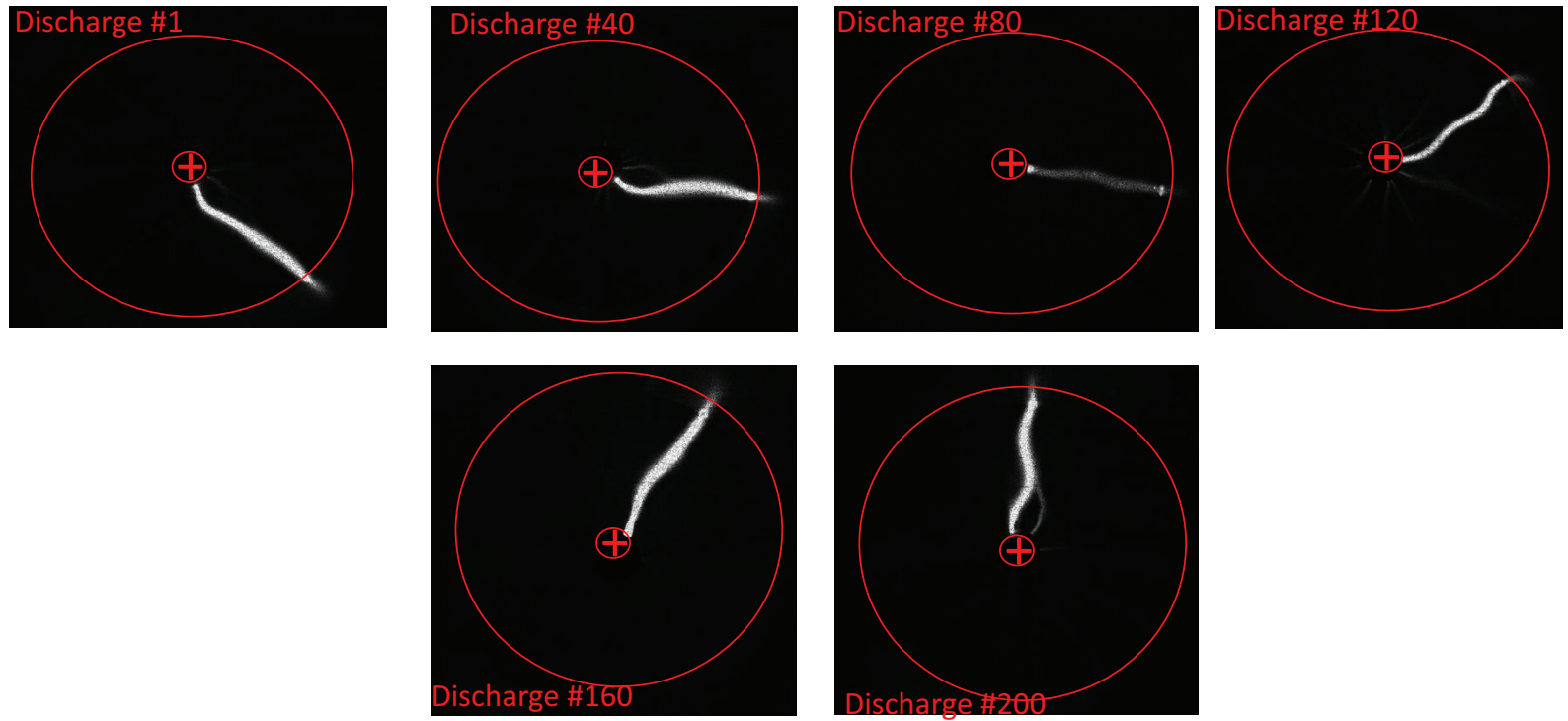

Figure 7. Spinning of positive polarity discharges recorded on a high speed camera at 10000 FPS

To summarize, at the lowest peak pulse voltage, the following observations can be made for negative polarity discharges where the central electrode is at negative potential and the images are shown in Figure 8:

- For low to moderate PRF (1-10 kHz), the discharge takes the mode of diffuse discharge channels spaced somewhat evenly around the electrode.

- As the PRF increases, the discharge channels become more constricted and their propagation distance also decreases.

- At low PRF $(1 \mathrm{kHz})$ and high PRF $(50 \mathrm{kHz})$, an increase in pulse duration does not appear to have much effect on the plasma; at moderate PRF $(10 \mathrm{kHz})$, increasing the pulse duration from 60 to 110 ns causes a significant increase in both plasma propagation distance and emission intensity.

- The discharge channels did not reach the outer electrode for any combination of pulse parameters.

While for the positive polarity discharges shown in Figure 9 where the central electrode is at a positive potential and a low input voltage of $14.6 \mathrm{kV}$ is supplied, the following observations can be made :

- For most of the pulse parameters, an arc is formed on the outer cylinder and tends to spin around at the pulse repetition frequency. This can be observed in the images from the high speed camera.

- At low PRF (1 kHz), one single arc is formed at a random spot for 40 and $60 \mathrm{ns,} \mathrm{while} \mathrm{for} \mathrm{the} \mathrm{high} \mathrm{pulse}$ width of 110 ns a higher number of arcs Coalesce over one half of the outer cylinder and propagate 
the entire distance between the two electrodes. However, there are also some tiny streamers on the opposite half which do not propagate the entire distance.

- At an intermediate PRF (10 kHz), there are a large number of constricted acrs for higher pulse widths, while for the low pulse width of $40 \mathrm{~ns}$, it's not highly constricted but there are a lot more streamers compared to PRF of $1 \mathrm{kHz}$. Unlike the low PRF, the tiny streamers are missing in this case.

- At high PRF of $50 \mathrm{kHz}$, the discharge is again to a random spot, but the discharge channels tend to twist and bend on their way to the cathode. This observation is true for all the pulse widths. With such twisting and bending, the flowfield induced by this discharge can be interesting to study as part of future work.

As the input voltage is increased to $16.7 \mathrm{kV}$, the negative polarity discharge behaves similar to the low voltage case with increase in emission intensity at $10 \mathrm{kHz}$ and constricted distinct streamers at $50 \mathrm{kHz}$. The only difference is at high PRF and pulse width of 60 and $110 \mathrm{~ns}$ where the discharge behaves similar to positive polarity discharge as shown in Figure 10. The positive polarity discharges on the other hand appear to be getting stronger at $1 \mathrm{kHz}$ and behave similar to the low voltage case at higher PRF as shown in Figure 11. A further increase in voltage to $18.75 \mathrm{kV}$ shows that the negative polarity discharge show the same structure as the low voltage case at low and intermediate PRF and behave same as positive polarity discharges at high PRF as shown in Figures 12 and 13. At the highest input voltage, the two polarity discharge have the same structure except at low pulse width of $40 \mathrm{~ns}$ and low to intermediate PRF of 1-10 $\mathrm{kHz}$ as shown in Figures 14 and 15.

Table 1. Pulse parameter combinations investigated in the current work. Each case in the matrix was tested using both positive and negative polarities on the center electrode.

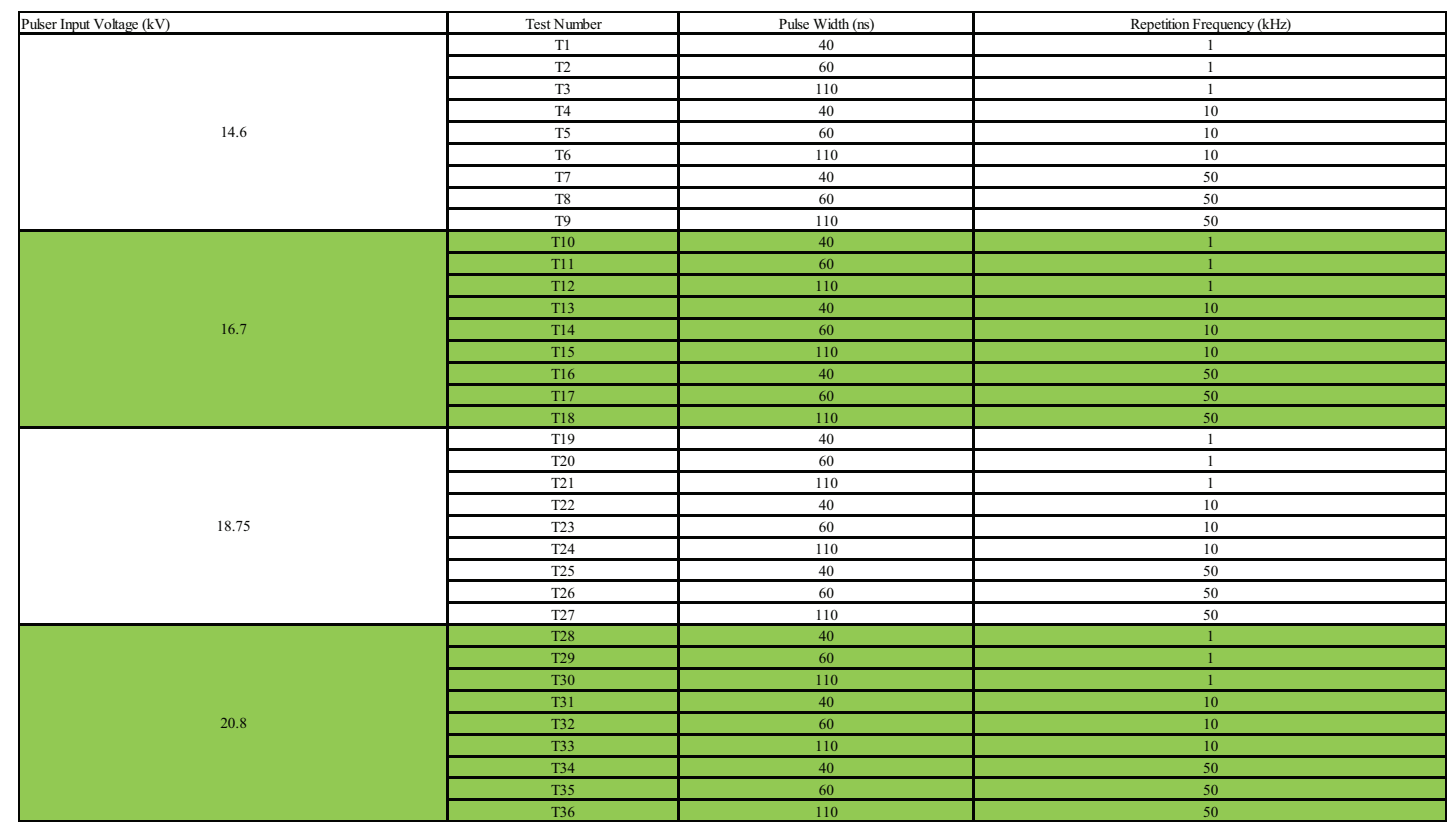

The peak breakdown voltage amplitude measured across the two electrodes is plotted for both cathode and anode driven discharges as shown in Figure 16. From the voltage plot it appears that the anode directed discharges require much higher breakdown voltages to form a uniform discharge compared to cathode directed discharge where an arc is formed. The breakdown voltages for anode directed streamers remain high until they transform to an arc from T29-T36, where the voltages for both cathode and anode directed discharges are comparable. The history effects due to high repetition rate is not significant in the case of anode directed streamers. Observing the ICCD images, the cathode directed streamers look more like a spark discharge with high current and the anode directed streamers appear like dielectric barrier discharge which is current limiting. This could possibly explain the difference in breakdown voltage. A current monitor will be used for the next phase of work to elaborate on this effect. A potential reason for having dielectric barrier discharge for anode directed streamers and spark like discharge for cathode directed streamer could be due to the 


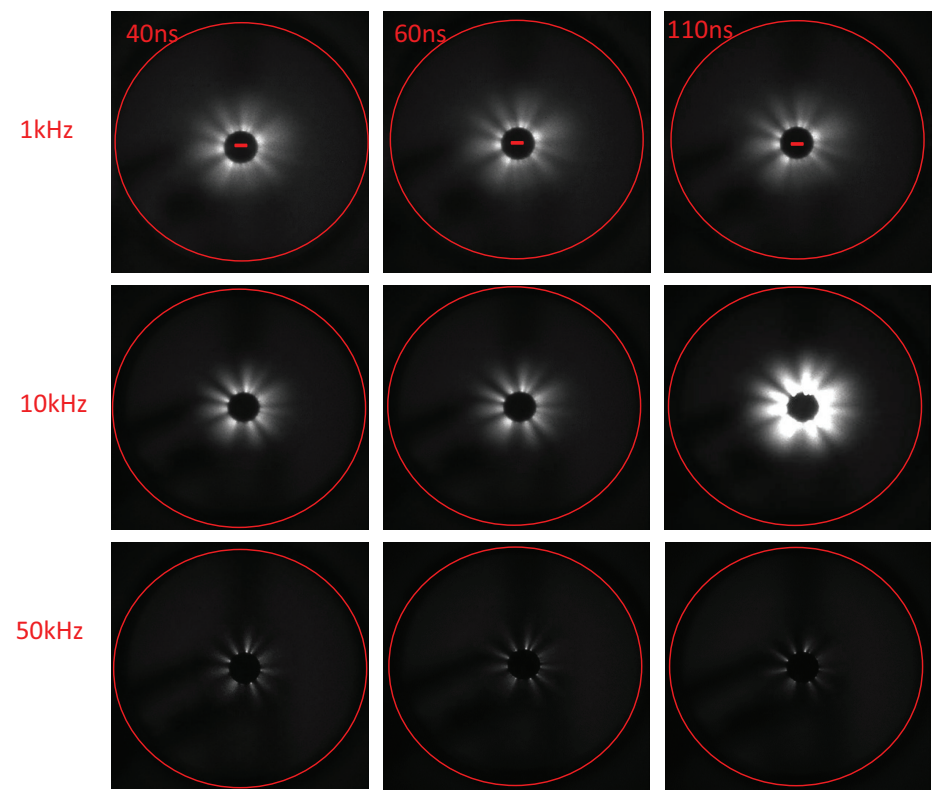

Figure 8. Negative polarity discharge with central electrode as cathode and pulser input voltage $=14.6 \mathrm{kV}$

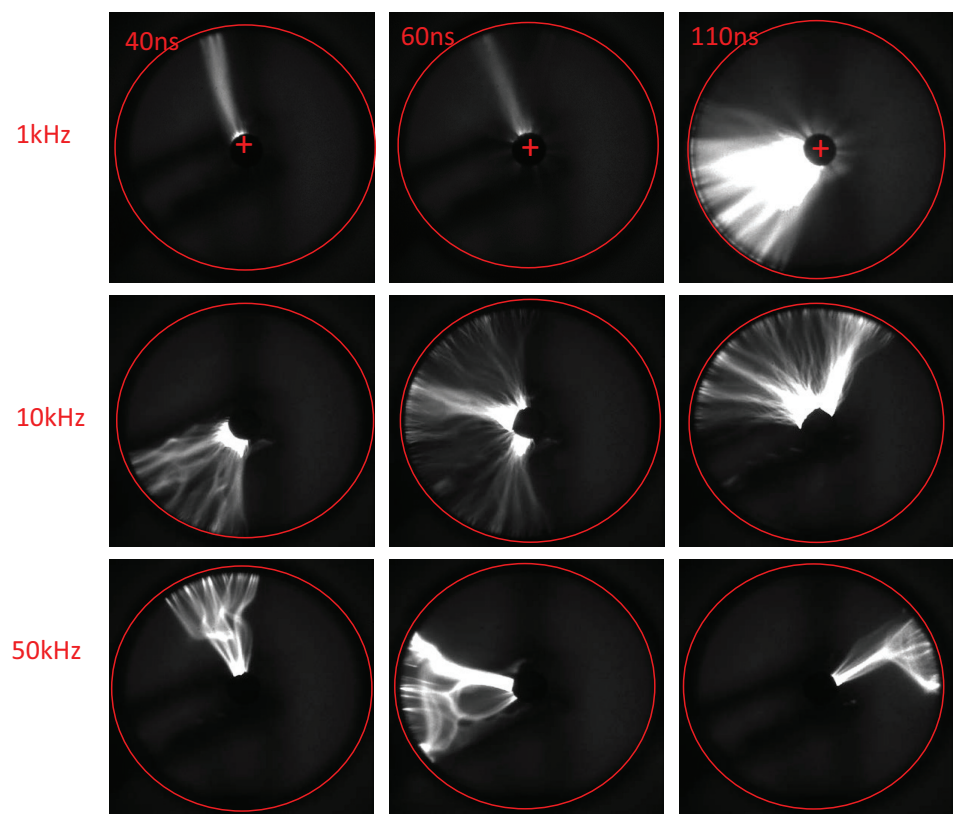

Figure 9. Positive polarity discharge with central electrode as anode and pulser input voltage $=14.6 \mathrm{kV}$ 


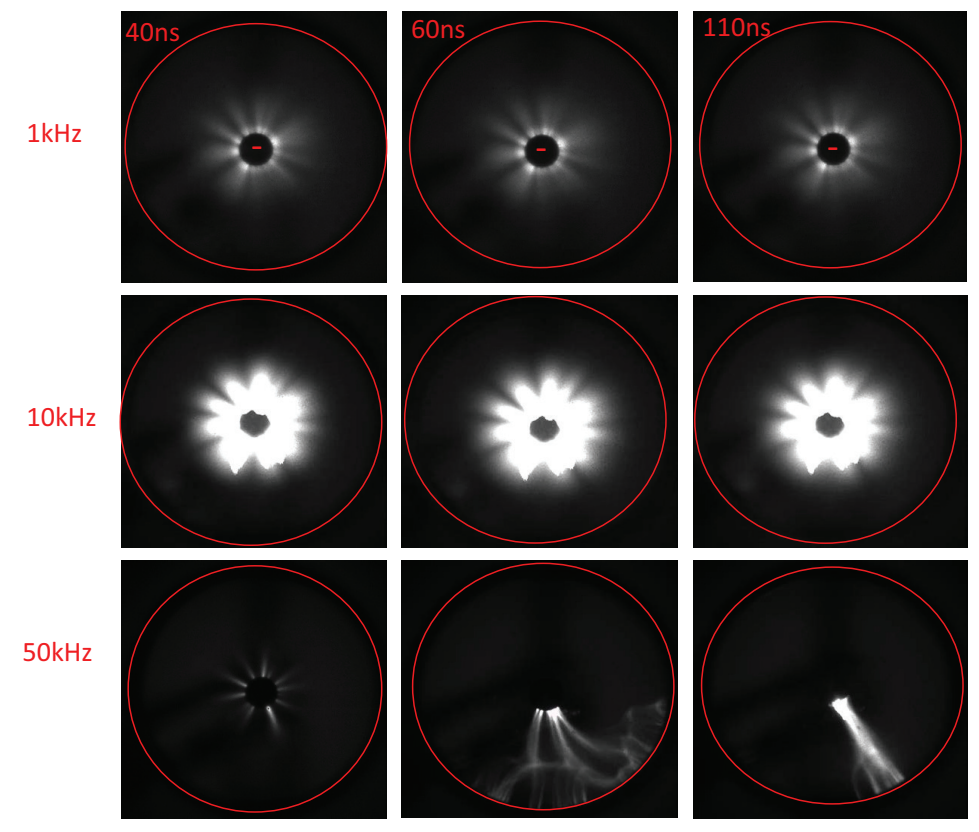

Figure 10. Negative polarity discharge with central electrode as cathode and pulser input voltage $=16.7 \mathrm{kV}$

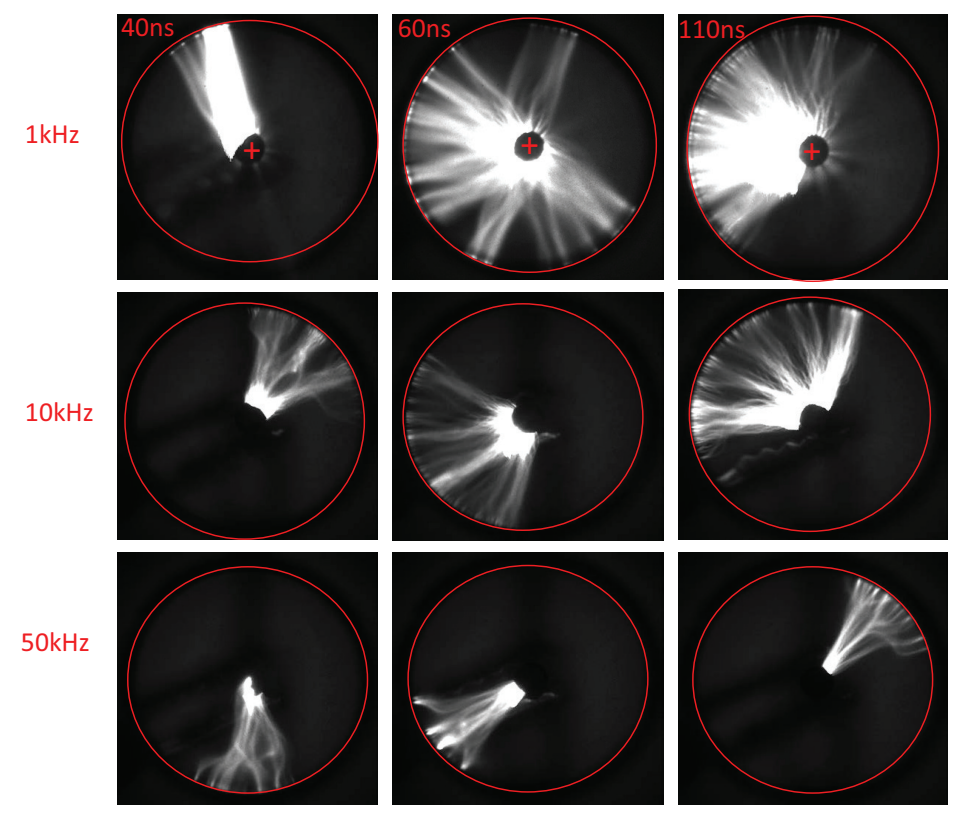

Figure 11. Positive polarity discharge with central electrode as anode and pulser input voltage $=16.7 \mathrm{kV}$ 


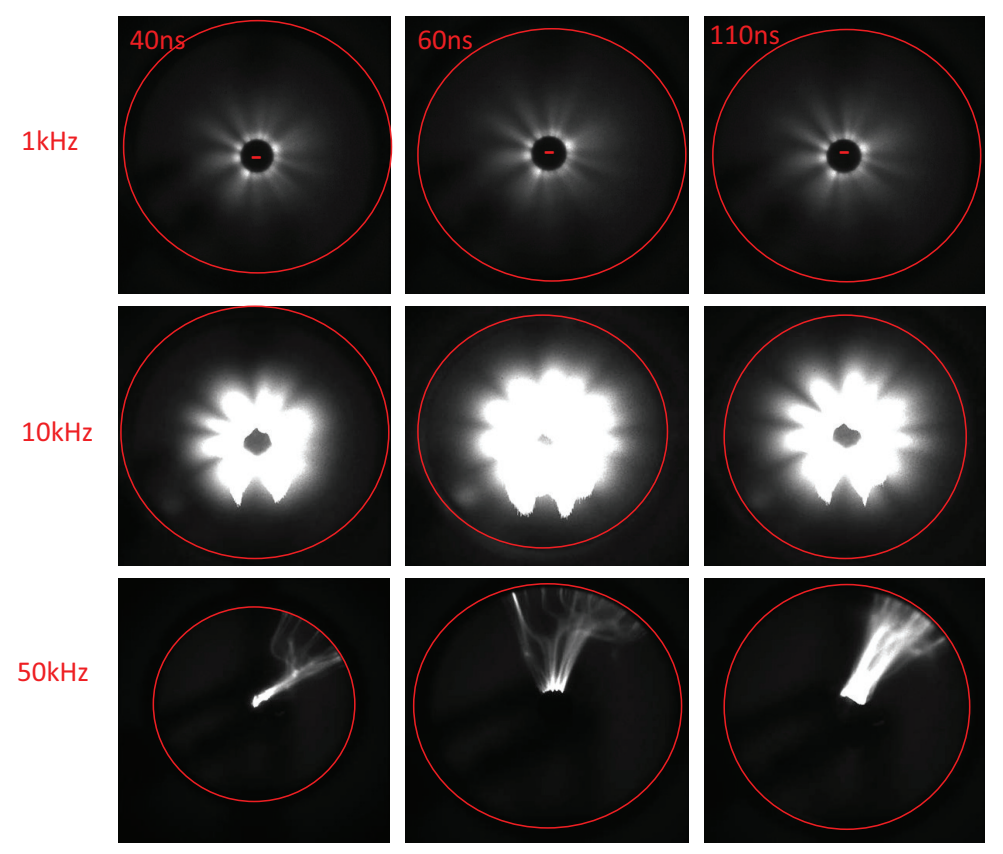

Figure 12. Negative polarity discharge with central electrode as cathode and pulser input voltage $=18.75 \mathrm{kV}$

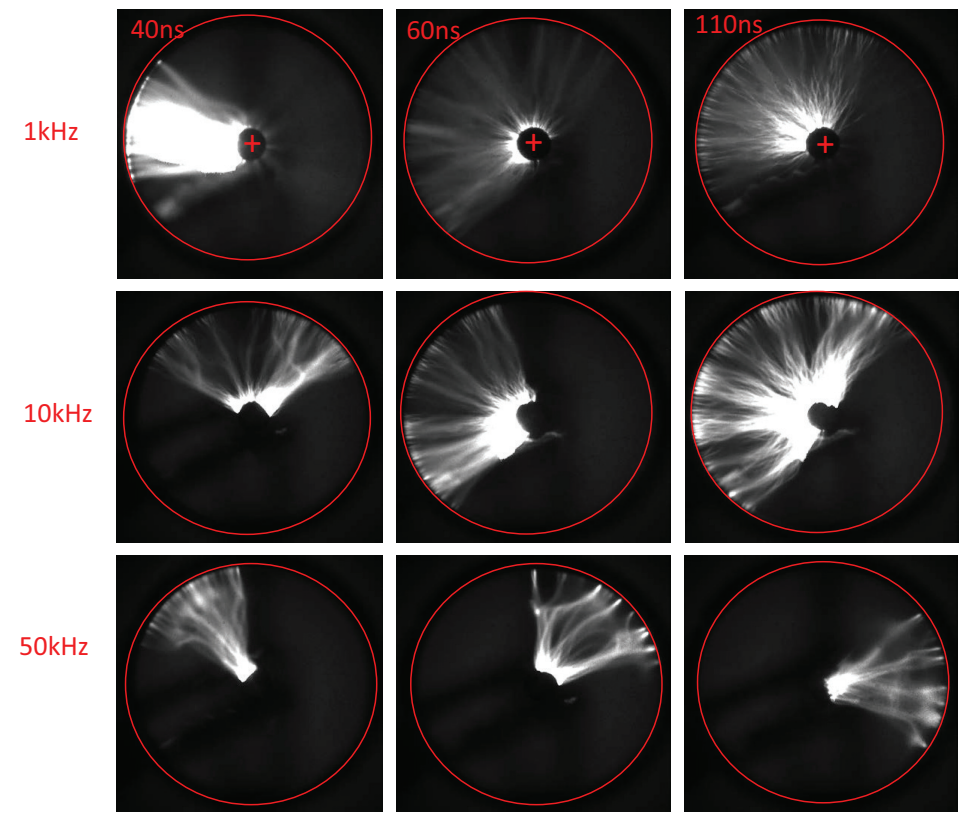

Figure 13. Positive polarity discharge with central electrode as anode and pulser input voltage $=18.75 \mathrm{kV}$ 

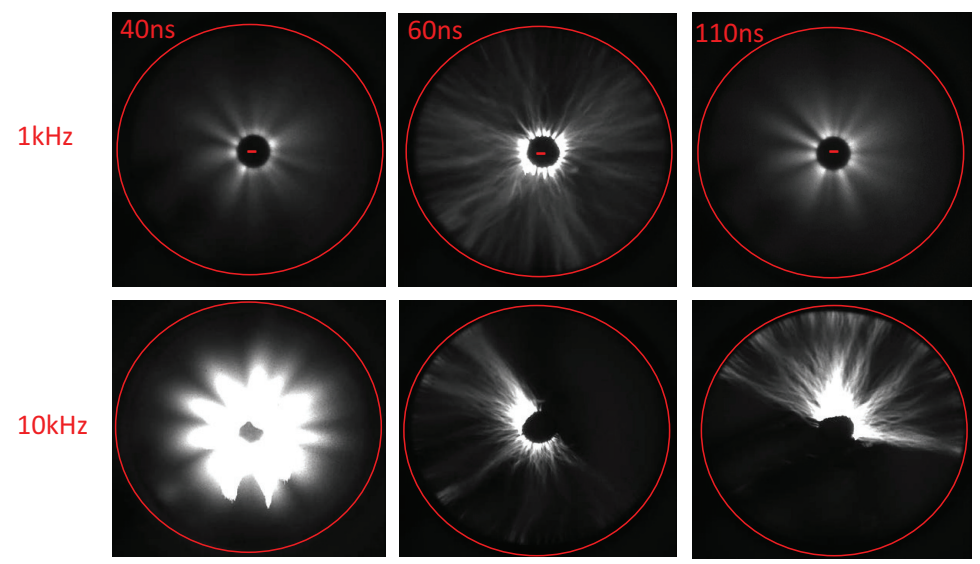

$50 \mathrm{kHz}$
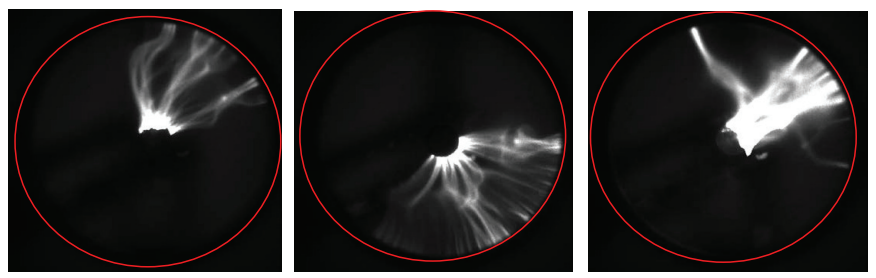

Figure 14. Negative polarity discharge with central electrode as cathode and pulser input voltage $=20.8 \mathrm{kV}$

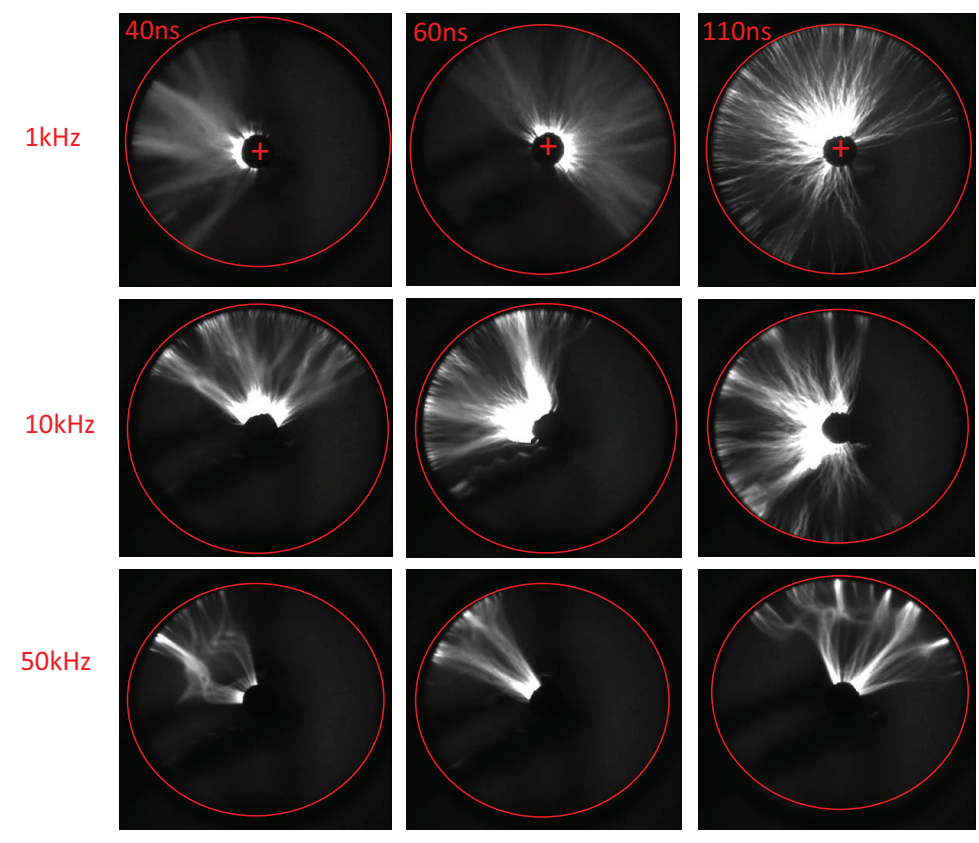

Figure 15. Positive polarity discharge with central electrode as anode and pulser input voltage $=20.8 \mathrm{kV}$ 
direction of ionization field and the input electric field. The electric field for a coaxial electrode is inversely proportional to the radial distance between the two electrodes as shown in Equation 1. From the equation, the electric field is maximum at the inner cylinder and decays as it moves out towards the outer cylinder. In the case of anode directed streamers, the input electric field is from the outer cylinder to the inner cylinder and is opposite to the ionization field. Since the two fields oppose each other, a much higher voltage is required to breakdown. Whereas in the case of cathode directed streamers, the input electric field is aligned from inner cylinder to the outer cylinder, this means the input field is in the same direction as the ionization field, therefore a lower voltage is required to breakdown. The observations from the images show a similar behavior observed for cathode and anode directed streamers in spark and dielectric barrier discharges. ${ }^{8,21,22}$

$$
E(r)=\frac{V_{o}}{r \ln \left(\frac{r_{\text {out }}}{r_{\text {in }}}\right)}
$$

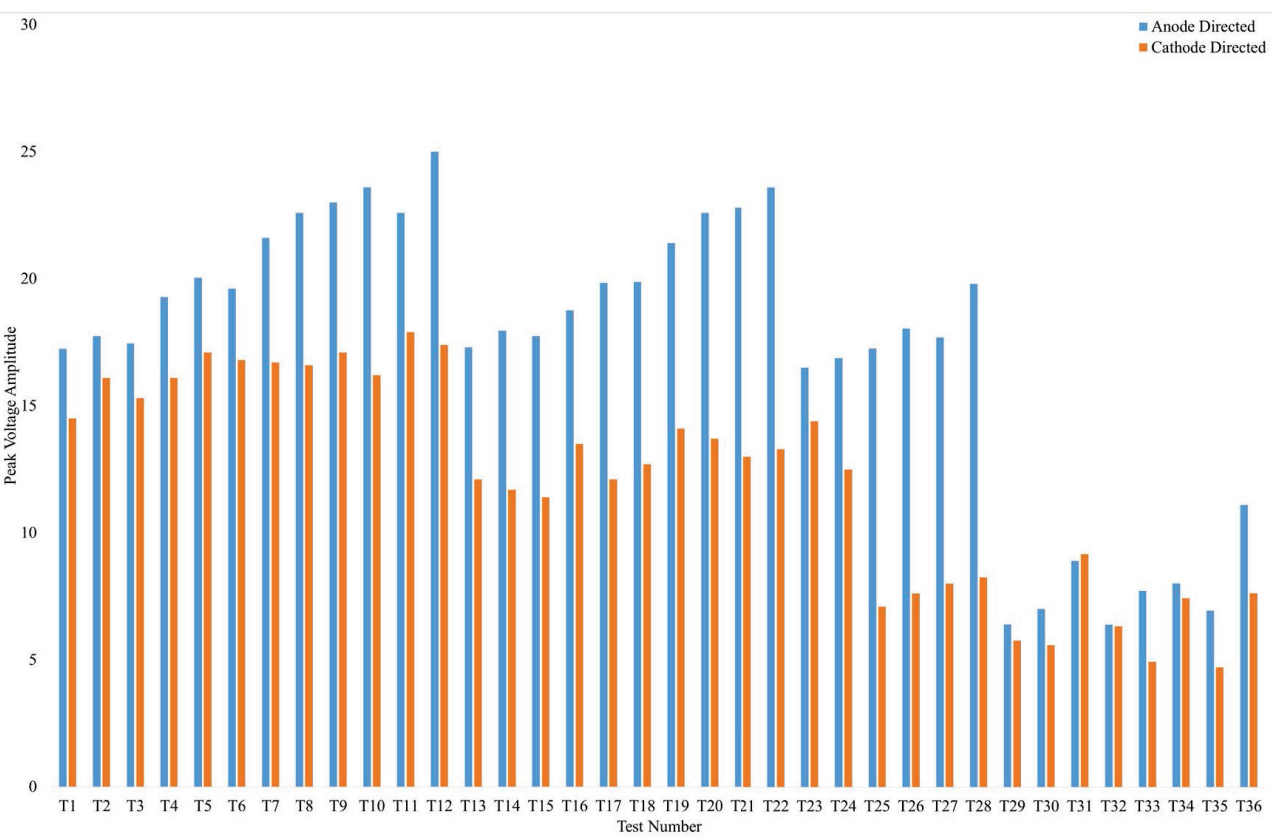

Figure 16. Voltage amplitude comparison between cathode and anode driven discharges

\section{Conclusion}

An experiment was developed to understand the development of plasma discharges in a coaxial electrode configuration. The current study is the first systematic investigation of discharges in a coaxial electrode configuration. The plasma formation was tested in quiescent, atmospheric and non-reactive environment. The plasma formation was visualized using an intensified ccd camera with 500 discharges in a single frame and high voltage probes were used to measure the breakdown voltage. The polarities of the central electrode was switched to visualize discharge formation with positive and negative polarity. Further, a high speed CMOS camera was used to confirm some of the findings with positive polarity discharges.

The iccd images show a distinct change between the positive and negative polarity discharges. When the central electrode is at negative polarity and low and intermediate PRFs the discharge appears quite uniform with diffuse discharge channels spaced at even intervals around the electrode circumference. At low PRFs and low input voltages, the pulse width appears to have no impact on discharge formation At the highest PRF $(50 \mathrm{kHz})$ the discharge channels form distinct streamers and both the plasma propagation distance and the emission intensity decrease drastically. As the voltage is increased, the negative polarity discharge transitions to an arc and behaves like the positive polarity discharge for a few pulse parameters. The positive polarity discharge on the other hand forms an arc discharge for most of the pulse parameters. At low PRF, it starts off as a single arc that spins about the axis and at higher PRFs, more number of arcs coalesce together 
at a random spot on the outer cylinder. The fact of spinning discharge was confirmed through the use of a high speed camera. The strong arc is seen rotating by more than $90 \circ$. The breakdown voltage provides some qualitative information. As the negative polarity transitions towards a positive polarity discharge, the breakdown voltages appear to be almost the same. When the negative polarity is having a uniform discharge, the breakdown voltage is much higher compared to positive polarity. A current probe in conjunction with voltage will provide a much better understanding of the discharge and will be reported in future investigation.

Further analysis is required to understand the difference between cathode and anode directed streamers and to provide an explanation regarding the unpredictable nature of positive polarity discharge. Different diagnostic tools will be required to analyze the behavior such as optical emission spectroscopy to estimate temperature and presence of different species and the use of microwave scattering to estimate changes in electron density. Different geometries will need to be analyzed for example the use of threaded rod for the inside cylinder and different diameter outer cylinder to design an optimal electrode geometry that can provide volumetric ignition. Future steps will include a much more detailed study of discharges in coaxial electrode in a non-reacting medium at different pressure, flow velocity and electrode axial gap before using it for ignition.

Volumetric ignition is achieved if the reactive mixture is ignited at multiple locations at the same time. The current study shows in the case of positive polarity discharges, one single arc is formed and it spins around the circumference. This may ignite the mixture at multiple spots as it spins around and may reduce ignition delay due to presence of active radicals and high electronic temperature of non-equilibrium plasmas, but it's no longer igniting at the same time. Whereas in the case of negative polarity discharges, the discharge is more uniform and the probability of igniting multiple spots at the same time is higher. Therefore, atleast from the initial imaging of discharges in a coaxial electrode, it seems more beneficial to have negative polarity discharges to achieve volumetric ignition. Experiments in reacting flow is required to see if it holds true. The current work has provided an initial understanding of the type of discharge in a coaxial electrode system and steps that can be taken during the next phase of work that can help in designing electrodes for plasma assisted volumetric ignition.

\section{References}

${ }^{1}$ Dec, J. E., "Advanced compression-ignition enginesunderstanding the in-cylinder processes," Proceedings of the combustion institute, Vol. 32, No. 2, 2009, pp. 2727-2742.

${ }^{2}$ Khan, M. N., Paik, K.-y., and Nalim, M. R., "3D computation for torch jet ignition of premixed methane-hydrogenair blends in a pre-chamber constant volume combustor at variable pre-chamber pressure," 51st AIAA/SAE/ASEE Joint Propulsion Conference, 2015, p. 3784.

${ }^{3}$ Shiraishi, T. and Urushihara, T., "Fundamental Analysis of Combustion Initiation Characteristics of Low Temperature Plasma Ignition for Internal Combustion Gasoline Engine," Tech. rep., SAE Technical Paper, 2011.

${ }^{4}$ Singleton, D., Pendleton, S., and Gundersen, M., "The role of non-thermal transient plasma for enhanced flame ignition in C2H4-air," Journal of Physics D: Applied Physics, Vol. 44, No. 2, 2010, pp. 022001.

${ }^{5}$ Pancheshnyi, S. V., Lacoste, D. A., Bourdon, A., and Laux, C. O., "Ignition of propane-air mixtures by a repetitively pulsed nanosecond discharge," IEEE Transactions on Plasma Science, Vol. 34, No. 6, 2006, pp. 2478-2487.

${ }^{6}$ Adamovich, I., Choi, I., Jiang, N., Kim, J., Keshav, S., Lempert, W., Mintusov, E., Nishihara, M., Samimy, M., and Uddi, M., "Plasma assisted ignition and high-speed flow control: non-thermal and thermal effects," Plasma Sources Science and Technology, Vol. 18, No. 3, 2009, pp. 034018.

${ }^{7}$ Corke, T. C., Enloe, C. L., and Wilkinson, S. P., "Dielectric barrier discharge plasma actuators for flow control*," Annual review of fluid mechanics, Vol. 42, 2010, pp. 505-529.

${ }^{8}$ Leonov, S. B., Adamovich, I. V., and Soloviev, V. R., "Dynamics of near-surface electric discharges and mechanisms of their interaction with the airflow," Plasma Sources Science and Technology, Vol. 25, No. 6, 2016, pp. 063001.

${ }^{9}$ Starikovskiy, A. and Aleksandrov, N., "Plasma-assisted ignition and combustion," Progress in Energy and Combustion Science, Vol. 39, No. 1, 2013, pp. 61-110.

${ }^{10}$ Shiraishi, T., Urushihara, T., and Gundersen, M., "A trial of ignition innovation of gasoline engine by nanosecond pulsed low temperature plasma ignition," Journal of Physics D: Applied Physics, Vol. 42, No. 13, 2009, pp. 135208.

${ }^{11}$ Popov, N., "Kinetics of plasma-assisted combustion: effect of non-equilibrium excitation on the ignition and oxidation of combustible mixtures," Plasma Sources Science and Technology, Vol. 25, No. 4, 2016, pp. 043002.

${ }^{12}$ Starikovskaia, S., "Plasma-assisted ignition and combustion: nanosecond discharges and development of kinetic mechanisms," Journal of Physics D: Applied Physics, Vol. 47, No. 35, 2014, pp. 353001.

${ }^{13}$ Wang, F., Liu, J., Sinibaldi, J., Brophy, C., Kuthi, A., Jiang, C., Ronney, P., and Gundersen, M. A., "Transient plasma ignition of quiescent and flowing air/fuel mixtures," IEEE Transactions on Plasma Science, Vol. 33, No. 2, 2005 , pp. 844-849.

${ }^{14}$ Starikovskiy, A., Rakitin, A., Correale, G., Nikipelov, A., Urushihara, T., and Shiraishi, T., "Ignition of hydrocarbon-air mixtures with non-equilibrium plasma at elevated pressures," Proc. of 50th AIAA Aerospace Sciences Meeting including the New Horizons Forum and Aerospace Exposition, 2012, pp. 9-12. 
${ }^{15}$ Boumehdi, M. A., Stepanyan, S. A., Desgroux, P., Vanhove, G., and Starikovskaia, S. M., "Ignition of methane-and n-butane-containing mixtures at high pressures by pulsed nanosecond discharge," Combustion and Flame, Vol. 162, No. 4, 2015, pp. 1336-1349.

${ }^{16}$ Lacoste, D., Moeck, J., Durox, D., Laux, C., and Schuller, T., "Effect of nanosecond repetitively pulsed discharges on the dynamics of a swirl-stabilized lean premixed flame," Journal of Engineering for Gas Turbines and Power, Vol. 135, No. 10, 2013, pp. 101501.

${ }^{17}$ Moeck, J. P., Lacoste, D. A., Durox, D., Guiberti, T. F., Schuller, T., and Laux, C. O., "Stabilization of a MethaneAir Swirl Flame by Rotating Nanosecond Spark Discharges," IEEE Transactions on Plasma Science, Vol. 42, No. 10, 2014, pp. 2412-2413.

${ }^{18}$ Heitz, S. A., Moeck, J. P., Schuller, T., Veynante, D., and Lacoste, D. A., "Influence of air flow parameters on nanosecond repetitively pulsed discharges in a pin-annular electrode configuration," Journal of Physics D: Applied Physics, Vol. 49, No. 15, 2016, pp. 155205.

${ }^{19}$ Shukla, B., Gururajan, V., Eisazadeh-Far, K., Windom, B., Singleton, D., Gundersen, M., and Egolfopoulos, F., "Effects of electrode geometry on transient plasma induced ignition," Journal of Physics D: Applied Physics, Vol. 46, No. 20, 2013, pp. 205201.

${ }^{20}$ Won, J. Y. and Williams, P., "Experimental study of streamers in pure N2 and N2/O2 mixtures and a 13 cm gap," Journal of Physics D: Applied Physics, Vol. 35, No. 3, 2002, pp. 205.

${ }^{21}$ Jiang, C., Chen, M., and Gundersen, M., "Polarity-induced asymmetric effects of nanosecond pulsed plasma jets," Journal of Physics D: Applied Physics, Vol. 42, No. 23, 2009, pp. 232002.

${ }^{22}$ Houpt, A. W. and Leonov, S. B., "Charge Transfer in Constricted Form of Surface Barrier Discharge at Atmospheric Pressure," Journal of Thermophysics and Heat Transfer, 2016, pp. 1-9. 